\title{
Instantaneous Bayesian regularization applied to real-time near-field acoustic holography
}

Thibaut Le Magueresse, Jean-Hugh Thomas, Jérôme Antoni, and Sébastien Paillasseur

Citation: The Journal of the Acoustical Society of America 142, 924 (2017); doi: 10.1121/1.4998571

View online: http://dx.doi.org/10.1121/1.4998571

View Table of Contents: http://asa.scitation.org/toc/jas/142/2

Published by the Acoustical Society of America

\section{Articles you may be interested in}

Beamforming using subspace estimation from a diagonally averaged sample covariance

The Journal of the Acoustical Society of America 142, 473 (2017); 10.1121/1.4995993

Wave Field Synthesis of moving sources with arbitrary trajectory and velocity profile

The Journal of the Acoustical Society of America 142, 551 (2017); 10.1121/1.4996126

A hybrid deconvolution approach to separate acoustic sources in multiple motion modes

The Journal of the Acoustical Society of America 142, 276 (2017); 10.1121/1.4994284

Removal of incoherent noise from an averaged cross-spectral matrix

The Journal of the Acoustical Society of America 142, 846 (2017); 10.1121/1.4997923

Two-dimensional grid-free compressive beamforming

The Journal of the Acoustical Society of America 142, 618 (2017); 10.1121/1.4996460

Space-time domain solutions of the wave equation by a non-singular boundary integral method and Fourier transform

The Journal of the Acoustical Society of America 142, 697 (2017); 10.1121/1.4996860 


\title{
Instantaneous Bayesian regularization applied to real-time near-field acoustic holography
}

\author{
Thibaut Le Magueresse ${ }^{a)}$ \\ MicrodB, 28 Chemin du Petit Bois, 69131 Ecully, France \\ Jean-Hugh Thomas \\ Laboratoire d'Acoustique de l'Université du Maine (LAUM), Unité Mixte de Recherche, Centre National de la \\ Recherche Scientifique 6613, Avenue O. Messiaen, 72085 Le Mans Cedex 09, France \\ Jérôme Antoni \\ Laboratoire Vibrations Acoustique, 25 bis Avenue Jean Capelle, 69621 Villeurbanne Cedex, France
}

Sébastien Paillasseur

MicrodB, 28 Chemin du Petit Bois, 69131 Ecully, France

(Received 11 December 2016; revised 2 July 2017; accepted 21 July 2017; published online 16 August 2017)

\begin{abstract}
Real-time near-field acoustic holography (RT-NAH) is used to recover non-stationary sound sources using a planar microphone array. Direct propagation is described by the convolution of the wavenumber spectrum of the source under study with a known impulse response. The deconvolution operation is achieved by a singular value decomposition of the propagator and Tikhonov regularization is performed to stabilize the solution. The inverse problem has an innate ill-posed characteristic, and the regularization process is the key factor in obtaining acceptable results. The purpose of this paper is to present the instantaneous regularization process applied to RT-NAH method. Bayesian estimation of the regularization parameter is introduced from prior knowledge of the problem. The computation of the regularization parameter is updated for each block of constant time interval allowing one to take into account the fluctuating properties of the sound field. The superiority of Bayesian regularization, compared to state-of-the art methods, is observed numerically and experimentally for reconstruction of non-stationary sources. RT-NAH is also enhanced to allow the reconstruction of long signals. Updating the regularization parameter accordingly to the fluctuations of the SNR is revealed to be a necessary effort to reconstruct highly non-stationary sources. (C) 2017 Acoustical Society of America.
\end{abstract}

[http://dx.doi.org/10.1121/1.4998571]

[ATW]

Pages: 924-934

\section{INTRODUCTION}

Near-field acoustical holography (NAH) is an effective experimental tool to reconstruct acoustic sources using discrete sound field measurements. ${ }^{1}$ Averaging time evolution over the whole acquisition duration makes it possible to work on converged cross spectra. However, most acoustic excitations have a non-stationary behavior: a car door slamming, pass-by noise, rotating sources, aeroacoustic noise, etc. Extensions of NAH have been proposed to address such cases. ${ }^{2-8}$ As standard NAH is usually computed for a single frequency and then devoted to stationary sources, "Time domain holography"2 was proposed for non-stationary sources: it consists of repeating NAH procedure on each spectral line and then applying the inverse Fourier transform to reconstruct a time evolution of sound field by an inverse Fourier transform. The process is well-adapted to short periods of time with limited bandwidth. A similar use of NAH has been suggested by Deblauwe et al. ${ }^{3}$ to track the time fluctuation of a particular frequency component. It aims to repeat the NAH process on time data blocks for a single frequency using short-time Fourier transform centered on the

${ }^{\text {a)} E l e c t r o n i c ~ m a i l: ~ t h i b a u t . l e-m a g u e r e s s e @ m i c r o d b . f r ~}$ frequency of interest. Both methodologies are based on the use of Fourier transforms in the time domain. Blais and Ross ${ }^{4}$ pointed out time aliasing discrepancies created by this process. The authors proposed then to implement time domain NAH using the numerical Laplace transform. ${ }^{5} \mathrm{Wu}$ et al. also used the Laplace transform to derive an analytical expression of the inverse propagator (so-called "temporal kernel") in the spherical coordinates and in the time domain. ${ }^{6}$ Finally, the estimated sound field radiated by the source is the result of the sound field measured by the array convolved with the kernel. Wu further explored this approach and recently proposed a novel method to characterize transient sources radiated by arbitrary shapes. ${ }^{7}$ The inverse propagator is determined in the frequency domain either by the boundary element method or by the Helmholtz equation least squares. With the aid of the residue theorem, the inverse Fourier transform is calculated to obtain the time kernels. Finally, Bai and $\operatorname{Lin}^{8}$ proposed a method named "near-field equivalence source imaging" based on the design of multichannel inverse filters to recover the strengths of non-stationary equivalent sources positioned near the source. These inverse filters can be interpreted as time kernels: once they are evaluated, the evolution of the sound field is obtained by a convolution with the measurements. 
For all of the aforementioned methods, the inverse problem appears to be ill-posed as the solution exhibits unstable behavior when the input data are perturbed by noise. Regularization methods have proved their efficiency to ensure the stability of the solution for standard NAH problems and non-stationary problems. ${ }^{9-11}$ The use of time kernels has the advantage of making possible the instantaneous estimation of the source field on the surface of interest, however, these approaches suffer from the same issue: the regularization process does not take into account the fluctuation of the energy of the acoustic signals. Statistical properties of the sound field are time-dependent and the amount of regularization has to be variable as well. Applying a constant filtering of singular values regardless of the instantaneous signal to noise ratio can entail significant errors of reconstruction. It seems that a time-dependent description of the acoustic radiation has to be provided with the aim of

- applying regularization process adapted to the fluctuation of signal energy relative to the time-varying noise energy;

- avoiding aliasing discrepancies produced by the inverse time Fourier transform.

Two strategies are available in the literature which depend on the domain of interest: the space-time domain for the first strategy and the time-wavenumber domain for the second. Both finally reconstruct the instantaneous sound field in the space domain.

The transient NAH based on time-domain equivalent source method operates directly in the time domain without applying any Fourier transform. ${ }^{12,13}$ Equivalent sources at each sample time are identified by solving an inverse problem. The proposed algorithm is by nature recursive and Tikhonov regularization has to be performed for each inverse problem resulting in a cumulative effect of errors. Reconstruction results have been enhanced by combining the method with the free-field time reversal. ${ }^{14} \mathrm{~A}$ similar iterative algorithm, called the time-domain plane wave superposition method (TD-PWSM), has been developed by Zhang et al. using an impulse response calculated in the timewavenumber domain. ${ }^{15,16}$ The decomposition into the plane waves basis allows both propagative and evanescent waves to be taken into account, which significantly improves the spatial resolution.

Real-time NAH (RT-NAH) also continuously reconstructs the time evolution of the acoustic field under study. ${ }^{17}$ A deconvolution problem is solved by using a discrete inverse response (different from that of TD-PWSM) in the timewavenumber domain. Unlike TD-PWSM, the method requires the computation of the wavenumber spectra in the measurement plane. In RT-NAH, the inverse problem is continuously solved for each point of the wavenumber domain independently thanks to the convolution product. For this reason, the RT-NAH calculation time is more attractive than the first strategy for industrial implementation. Furthermore, RT-NAH does not use the equivalent source idea, exploited in TDPWSM, that consists of representing the sound field with a finite number of monopoles positioned on a virtual distance from the source plane. The position of the virtual source is an input parameter which significantly influences the results. Optimal choice of the retreat distance is not known yet and only empirical hints are so far available in the literature. ${ }^{18}$

Both strategies offer a unique insight into instantaneous reconstruction of acoustic radiation. Most importantly, the reconstruction error of these methods is limited by the use of a time-dependent regularization. Empirical Bayesian regularization applied to acoustic inverse problems has been introduced by Antoni. ${ }^{19}$ In the case where additive noise source distributions are supposed to be Gaussian, the Bayesian approach leads naturally to the general-form Tikhonov solution obtained from the knowledge of the propagation model, the measurements, and the regularization parameter. This parameter is calculated by making a compromise between the fidelity to the real measured data and the fidelity to available a priori information. Optimally tuning the regularization parameter arises as a major issue in determining the quality of the reconstruction. Some specific methods ${ }^{20,21}$ have been developed to automatically evaluate the parameter. The L-curve (LCV) and the generalized cross validation (GCV), among others, seem to prevail in the vibro-acoustic field. The Bayesian approach (BA) also offers a solution for estimating the regularization parameter. Its superiority compared to the state-of-the-art methods (GCV and LCV) has been proven in stationary inverse problems. ${ }^{22}$

The aim of this paper is to evaluate the performance of instantaneous Bayesian regularization applied to the reconstruction of long non-stationary sources using RT-NAH. Moreover, the interest in updating the regularization parameter per block over time is highlighted.

\section{FORWARD PROPAGATION IN THE TIME-WAVENUMBER DOMAIN}

Let us consider the acoustic propagation between a calculation plane $z_{c}$ and a measurement plane $z_{m}$ generated by a non-stationary source placed at $z_{s}$ as sketched in Fig. 1 .

The sound pressure is decomposed into plane waves using the two-dimensional Fourier transform along $x$ and $y$ directions,

$$
P\left(k_{x}, k_{y}, z, t\right)=\int_{-\infty}^{+\infty} \int_{-\infty}^{+\infty} p(x, y, z, t) e^{j\left(k_{x} x+k_{y} y\right)} d x d y
$$

with $k_{x}$ and $k_{y}$ the wavenumbers along the $x$ axis and $\mathrm{y}$ axis, respectively. The acoustic propagation in the time-wavenumber domain is expressed as a time-convolution between the wavenumber spectrum of sources at the calculation plane, $P\left(k_{x}, k_{y}, z_{c}, t\right)$, and the impulse response $h\left(k_{x}, k_{y}, \Delta z, t\right)$,

$$
\begin{aligned}
P\left(k_{x}, k_{y}, z_{m}, t\right)= & P\left(k_{x}, k_{y}, z_{c}, t\right) *_{t} h\left(k_{x}, k_{y}, \Delta z, t\right) \\
& +B\left(k_{x}, k_{y}, t\right),
\end{aligned}
$$

with $P\left(k_{x}, k_{y}, z_{m}, t\right)$ being the instantaneous wavenumber spectrum measured at the measurement plane, $B\left(k_{x}, k_{y}, t\right)$ the additive noise, $\Delta z=z_{m}-z_{c}$ the distance of back propagation, and $*_{t}$ the convolution operator in time. The impulse response is obtained as the solution of the Helmholtz equation in the time-wavenumber domain, 


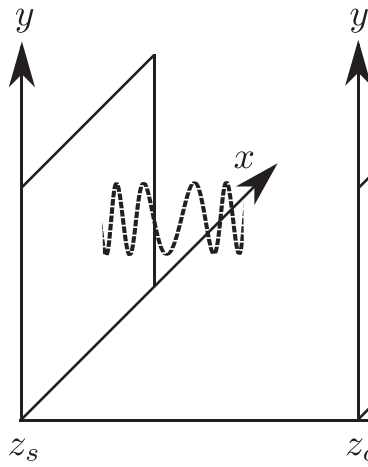

$$
\begin{gathered}
\frac{\partial^{2} P\left(k_{x}, k_{y}, z, t\right)}{\partial z^{2}}-\frac{1}{c^{2}} \frac{\partial^{2} P\left(k_{x}, k_{y}, z, t\right)}{\partial t^{2}} \\
-\left(k_{x}^{2}+k_{y}^{2}\right) P\left(k_{x}, k_{y}, z, t\right)=0,
\end{gathered}
$$

with $c$ being the celerity of the acoustical waves and $P\left(k_{x}, k_{y}, z, t\right)=0$ for $t<\tau$, where $\tau=(\Delta z) / c$ is the propagation delay. The derivation of this equation leads to an analytical expression of the impulse response function, ${ }^{23,24}$

$$
h\left(k_{x}, k_{y}, \Delta z, t\right)=h\left(\Omega_{r}, \tau, t\right)=\delta(t-\tau)-g\left(\Omega_{r}, \tau, t\right),
$$

where $\delta(\tau)$ is the Dirac function, $\Omega_{r}=c \sqrt{k_{x}^{2}+k_{y}^{2}}$ is the transition angular frequency, and

$$
g\left(\Omega_{r}, \tau, t\right)=\tau \Omega_{r}^{2} \frac{J_{1}\left(\Omega_{r} \sqrt{t^{2}-\tau^{2}}\right)}{\Omega_{r} \sqrt{t^{2}-\tau^{2}}} \Gamma(t-\tau),
$$

with $J_{1}$ being the Bessel function of the first kind of order 1 and where $\Gamma(t)$ is the Heaviside function.

In practice, the measured sound field is regularly sampled at time instants $t=n \Delta t, n=1,2, \ldots, N$ and the direct problem is reformulated as

$$
\begin{aligned}
P\left(k_{x}, k_{y}, z_{m}, n \Delta t\right)= & P\left(k_{x}, k_{y}, z_{c}, n \Delta t\right) *_{t} h\left(k_{x}, k_{y}, \Delta z, n \Delta t\right) \\
& +B\left(k_{x}, k_{y}, n \Delta t\right)
\end{aligned}
$$

FIG. 1. Sketch illustrating the geometry of interest. where $h(\ldots, n \Delta t)$ stands for the discretized version of the impulse response $h(\ldots, t)$. Sampling the impulse response causes phase and magnitude discrepancies, as mentioned by Grulier et al. ${ }^{24}$ They proposed corrections based on designing low-pass filters to overcome these difficulties by comparing the theoretical transfer function with the discrete Fourier transform of the impulse response. Another solution recommended ${ }^{17,24}$ to reduce such errors is to replace direct sampling by average sampling, for instance, by using the trapezoidal formula

$$
\bar{g}[n]=g\left(\Omega_{r}, \tau, n\right)=\frac{1}{\Delta t} \int_{n \Delta t-\Delta t / 2}^{n \Delta t+\Delta t / 2} g\left(\Omega_{r}, \tau, t\right) d t .
$$

Due to the property of the impulse response, the forward problem can be written as a discrete sum,

$$
\begin{aligned}
P\left(z_{m}, n \Delta t\right)= & \sum_{m=0}^{n} P\left(z_{c}, m \Delta t\right) h\left(k_{x}, k_{y}, \Delta z, n \Delta t-m \Delta t\right) \\
& +B(n \Delta t) .
\end{aligned}
$$

In addition, from Eq. (5), the impulse response is equal to zero for $t<\tau$, corresponding to the time needed by the waves to propagate from the calculation plane to the measurement plane. Considering $n_{\tau}$ the smallest sample for which $\tau \leq n_{\tau} \times T_{s}$, with $T_{s}$ the sampling period, the forward problem of Eq. (8) is expressed as a classical linear problem,

$$
\left[\begin{array}{c}
P_{z_{m}}\left(n_{\tau}\right) \\
\vdots \\
\vdots \\
\vdots \\
P_{z_{m}}(N-1)
\end{array}\right]=\left[\begin{array}{ccccc}
h_{\Delta z}\left(n_{\tau}\right) & 0 & \cdots & \cdots & 0 \\
\vdots & \ddots & \ddots & & \vdots \\
\vdots & & \ddots & \ddots & \vdots \\
\vdots & & & \ddots & 0 \\
h_{\Delta z}(N-1) & \cdots & \cdots & \cdots & h_{\Delta z}\left(n_{\tau}\right)
\end{array}\right]\left[\begin{array}{c}
P_{z_{c}}(0) \\
\vdots \\
\vdots \\
\vdots \\
P_{z_{c}}\left(N-n_{\tau}\right)
\end{array}\right]+\left[\begin{array}{c}
B(0) \\
\vdots \\
\vdots \\
\vdots \\
B\left(N-n_{\tau}\right)
\end{array}\right] .
$$

Using matrix formalism, Eq. (9) reads

$$
\mathbf{P}_{z_{m}}=\mathbf{H}_{\Delta z} \mathbf{P}_{z_{c}}+\mathbf{B}
$$

with $\mathbf{P}_{z_{m}}$ and $\mathbf{P}_{z_{c}}$ being the instantaneous wavenumber spectra at the measurement plane and the calculation plane, respectively, and $\mathbf{H}_{\Delta z}$ a Toeplitz matrix.

\section{BA TO DECONVOLUTION}

\section{A. Probabilistic formalism}

The aim of the deconvolution is to reconstruct the wavenumber spectra $\mathbf{P}_{z_{c}}$ from the measured wavenumber spectra $\mathbf{P}_{z_{m}}$. If we consider the wavenumber spectra as random variables, the goal consists of finding the conditional probability 
density function (pdf) of $\mathbf{P}_{z_{c}}$ given $\mathbf{P}_{z_{m}}$, denoted by $\left[\mathbf{P}_{z_{c}} \mid \mathbf{P}_{z_{m}}\right]$. This pdf is unknown but can be estimated according to Bayes' rule which expresses the posterior probability as

$$
\left[\mathbf{P}_{z_{c}} \mid \mathbf{P}_{z_{m}}\right]=\frac{\left[\mathbf{P}_{z_{m}} \mid \mathbf{P}_{z_{c}}\right]\left[\mathbf{P}_{z_{c}}\right]}{\left[\mathbf{P}_{z_{m}}\right]}
$$

where $[X]$ represents the probability density function of random variable $X$ and $[X \mid Y]$ the conditional probability of $X$ given $Y$. The density $\left[\mathbf{P}_{z_{m}} \mid \mathbf{P}_{z_{c}}\right]$ represents the likelihood function, i.e., the pdf of the measurements $\mathbf{P}_{z_{m}}$ knowing the instantaneous wavenumber spectrum of the source $\mathbf{P}_{z_{c}}$. The prior $\left[\mathbf{P}_{z_{c}}\right]$ is the pdf of the sources before observing the measurements. Finally, the quantity $\left[\mathbf{P}_{z_{m}}\right]$ expresses the pdf of the measurements for all possible sources considered.

\section{Modeling the probability density functions}

Experimental errors have many different origins and, as a consequence of the central limit theorem, they are well modelled by a Gaussian pdf. $^{25}$ In the timewavenumber domain where the wavenumber spectrum is a complex value, additive noise then follows a complex zero-mean Gaussian law $\mathcal{N}_{c}\left(0, \beta^{2} \Omega_{\boldsymbol{b}}\right)$ where $\beta^{2}$ stands for the unknown energy of noise and $\Omega_{b}$ is a known structure matrix defining the nature of the noise (an identity matrix is usually chosen to enforce zero correlation of noise between microphones):

$$
\left[\mathbf{B} \mid \beta^{2}\right]=\mathcal{N}_{c}\left(0, \beta^{2} \Omega_{b}\right)=\frac{1}{\pi^{N} \beta^{2 N}\left|\Omega_{b}\right|} \exp \left(-\frac{\|\mathbf{B}\|_{\Omega_{b}}^{2}}{\beta^{2}}\right),
$$

where $|\mathbf{X}|$ denotes the determinant of matrix $\mathbf{X}$ and the notation $\|\mathbf{X}\|_{\mathbf{Y}_{x}}^{2}=\mathbf{X}^{H} \mathbf{Y}_{x}^{-1} \mathbf{X}$ is used.

The likelihood function $\left[\mathbf{P}_{z_{m}} \mid \mathbf{P}_{z_{c}}\right]$ is derived using the direct problem expressed in Eq. (10). Since measurements are perturbed by the additive noise defined above, $\mathbf{P}_{z_{m}}$ conditionally follows a complex Gaussian law with mean $\mathbf{H}_{\Delta z} \mathbf{P}_{z_{c}}$ and covariance matrix $\beta^{2} \Omega_{b}$,

$$
\left[\mathbf{P}_{z_{m}} \mid \mathbf{P}_{z_{c}}, \beta^{2}\right]=\mathcal{N}\left(\mathbf{H}_{\Delta z} \mathbf{P}_{z_{c}}, \beta^{2} \Omega_{b}\right)
$$

Last, prior information about the nature of sound sources in the time-wavenumber domain is also chosen as a Gaussian distribution. This hypothesis is mathematically convenient even if other choices could be adopted; ${ }^{26}$ however, these will not be investigated in this paper. The prior probability density function of sources thus reads

$$
\left[\mathbf{P}_{z_{c}} \mid \alpha^{2}\right]=\mathcal{N}_{c}\left(0, \alpha^{2}\right)=\frac{1}{\pi^{N} \alpha^{2 N}\left|\Omega_{s}\right|} \exp \left(-\frac{\left\|\mathbf{P}_{z_{c}}\right\|_{\Omega_{s}}^{2}}{\alpha^{2}}\right),
$$

with $\alpha^{2}$ standing for the unknown energy of sources and $\Omega_{s}$ being a known structure matrix defining time correlation of sources, also chosen as the identity matrix in the current study.

\section{B. Maximum a posteriori estimation}

The most probable solution of the inverse problem is obtained by determining the maximum a posteriori estimator. This value is given by maximizing the numerator in Eq. (11), or alternatively by minimizing the opposite of its logarithm which is easier to manipulate. This leads to the following cost function:

$$
J=-\ln \left[\mathbf{P}_{z_{c}}, \alpha^{2}, \beta^{2} \mid \mathbf{P}_{z_{m}}\right]
$$

Enforcing the derivative of this cost function to zero with respect to the solution $\mathbf{P}_{z_{c}}$ brings us to an analytical expression of the sources,

$$
\mathbf{P}_{z_{c}}^{\eta}=\mathbf{V}\left\lceil\frac{\mathbf{s}}{\mathbf{s}^{2}+\eta^{2}}\right\rfloor \mathbf{U}^{H} \mathbf{P}_{z_{m}}=\mathbf{H}_{\Delta z}^{\dagger}\left(\eta^{2}\right) \mathbf{P}_{z_{m}}
$$

where $\mathbf{V}$ and $\mathbf{U}$ are the singular orthonormal matrices, $\mathbf{s}$ is a diagonal matrix containing singular values stemming from the singular values decomposition of the Toeplitz-form matrix $\mathbf{H}_{\Delta z}=\mathbf{U}\lceil\mathbf{s}\rfloor \mathbf{V}^{H}, \eta^{2}=\beta^{2} / \alpha^{2}$ is the regularization parameter, and $\mathbf{H}_{\Delta z}^{\dagger}\left(\eta^{2}\right)$ is the inverse and regularized form of the matrix $\mathbf{H}_{\Delta z}$. It is noteworthy that this solution is similar to the Tikhonov solution. In addition, regularization is applied here only once from the overall vector $\mathbf{P}_{z_{m}}$ containing the measured time-wavenumber spectrum.

\section{Empirical Bayesian regularization}

In the empirical Bayesian framework, the regularization parameter is determined by maximizing the posterior probability density function $\left[\eta^{2} \mid \mathbf{P}_{z_{m}}\right]$ marginalized with respect to $\mathbf{P}_{z_{c}}$, i.e., ${ }^{27}$

$$
\begin{aligned}
{\left[\eta^{2} \mid \mathbf{P}_{z_{m}}\right] } & =\int\left[\eta^{2} \mid \mathbf{P}_{z_{m}}, \mathbf{P}_{z_{c}}\right]\left[\mathbf{P}_{z_{c}}\right] d \mathbf{P}_{z_{c}} \\
& \propto\left(\left(\sum_{k=1}^{M} \frac{\left|\mathbf{U}_{k}^{H} \mathbf{P}_{z_{m}}\right|^{2}}{s_{k}^{2}+\eta^{2}}\right)^{M-2} \prod_{k=1}^{M}\left(s_{k}^{2}+\eta^{2}\right)\right),
\end{aligned}
$$

where $M$ is the number of microphones mounted in the array. An estimation of the regularization parameter is then determined by the minimization of the cost function,

$$
\begin{aligned}
\eta_{M A P}^{2} & =\underset{\eta^{2}}{\operatorname{Argmin}}\left(-\ln \left[\eta^{2} \mid \mathbf{P}_{z_{m}}\right]\right) \\
& =\underset{\eta^{2}}{\operatorname{Argmin}}\left(J_{2}\left(\eta^{2}\right)\right)
\end{aligned}
$$

with

$$
J_{2}\left(\eta^{2}\right)=\sum_{k=1}^{M} \ln \left(s_{k}^{2}+\eta^{2}\right)+(M-2) \ln \alpha^{2}
$$

and

$$
\alpha^{2}\left(\eta^{2}\right)=\frac{1}{M} \sum_{k=1}^{M} \frac{\left|\mathbf{U}_{k}^{H} \mathbf{P}_{z_{m}}\right|^{2}}{s_{k}^{2}+\eta^{2}}
$$


The second hyper-parameter is also estimated as $\beta^{2}=\alpha^{2} \times \eta^{2}$.

It is remarkable that the implementation of this regularization process is extremely simple as it boils down to the search of a minimum of a 1D cost function.

\section{Batch deconvolution}

In RT-NAH, a global regularization strategy is faced with two difficulties. First, deconvolution has to be performed from Eq. (10) where the squared-matrix $\mathbf{H}_{\Delta z}$ has dimensions $\left(N-n_{\tau}\right) \times\left(N-n_{\tau}\right)$, with $N-n_{\tau}$ the total number of samples to be reconstructed. If the non-stationary phenomenon is characterized by a long duration (approximatively a second for example) sampled at Fs $=16 \mathrm{kHz}$ for instance, the storage and calculation capabilities of the computer have to be considerable. Second, the regularization of the singular values is applied to the overall duration of the signal. In situations where short non-stationarities occur, a global regularization strategy is generally not optimal since the regularization parameter is itself likely to evolve as a function of time. With this perspective in mind, the inverse process has to be applied many times on shorter blocks in order to (1) limit the size of the matrix to invert and (2) update the regularization parameter value.

The batch deconvolution will be computed iteratively on $K$ blocks of $L$ samples, each with $K L=N$, where $L$ corresponds to the impulse response length; the latter has to be chosen sufficiently large to accurately describe the decay of the impulse response. Time blocks of $L$ samples of the instantaneous wavenumber spectrum are processed iteratively. In order to avoid aliasing problems due to the discontinuities introduced at the edges of the truncated intervals, the dimension of the problem is expanded: $M$ zeros are introduced at the beginning and at the end of the blocks. Likewise, the Toeplitz matrix $\mathbf{H}_{\Delta z}$ of Eq. (10) is upgraded to dimensions $(2 M+L) \times(2 M+L)$. For one block of data, the direct problem reads

$$
\left[\begin{array}{c}
0 \\
\vdots \\
\frac{P_{z_{m}}\left(n_{\tau}\right)}{\vdots} \\
\frac{P_{z_{m}}(L-1)}{0} \\
\vdots \\
0
\end{array}\right]=\left[\begin{array}{l}
h\left(n_{\tau}\right) \\
\vdots \\
h(L-1) \\
0 \\
\vdots \\
0
\end{array}\right.
$$$$
\begin{array}{ll}
0 & \\
& \\
& 0 \\
& h\left(n_{\tau}\right) \\
& \vdots \\
0 & h(L-1) \\
& \\
& \\
& \ldots
\end{array}
$$
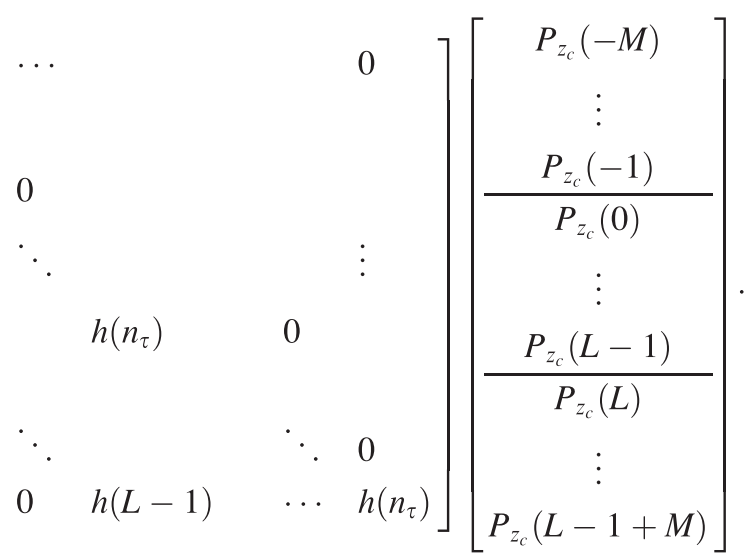

The solutions obtained by inverting system (23) for each block of data are then combined by summation: the $M$ first samples are added to the previous block and the last ones are added to the next block. Moreover, a Hanning windowing is applied to the measured vector insuring a $50 \%$ of overlap. Figure 2 depicts the procedure. Note that this procedure is exact; the only possible source of error comes from the truncation of the impulse response $h$ to $L$ samples.

\section{NUMERICAL VALIDATIONS}

A numerical study has been conducted to evaluate the performance of BA regularization in RT-NAH as compared with the state-of-the-art methods, namely LCV and GCV. Similar studies have been conducted in the case of reconstruction of stationary sources. ${ }^{28,29}$

\section{A. Numerical set-up}

The numerical procedure involves the propagation of a point source situated in the source plane at $z_{s}$ to the calculation plane at $z_{c}$ under a free-field hypothesis in the time domain. The point source is positioned at $(0.2,0.4,0)$ $\mathrm{m}$ and is defined by a signal with a linear frequency modulation in the band $[300 \mathrm{~Hz}, 3000 \mathrm{~Hz}]$ and a Gaussian amplitude modulation. The signal is sampled at $\mathrm{Fs}=16 \mathrm{kHz}$, thus providing 256 samples in a time snapshot of $16 \mathrm{~ms}$ centered on $s(t)$. The sound pressure is propagated to 49 points distributed evenly in the space by a step of $0.1 \mathrm{~m}$. The propagation from the calculation plane to the measurement plane is carried out by the convolution of the field at $z_{c}=0.01 \mathrm{~m}$ and the impulse response function according to Eq. (8).

Two indicators are introduced to assess the reconstruction results in an objective manner. They characterize, in the space domain, the accuracy of the time sound fields $p\left(x, y, z_{c}, t\right)$ reconstructed on the calculation plane by applying the inverse two-dimensional spatial Fourier transform, for each time instant, to the time wavenumber spectrum established from the components of $\mathbf{P}_{\mathbf{z}_{\mathbf{c}}}$ for each wavenumber pair $\left(k_{x}, k_{y}\right)$. The indicators represent the quality of the phase and the magnitude reconstruction and are, respectively, noted $T_{1}$ and $T_{2}$ for each point $i, j$ of the spatial domain, 


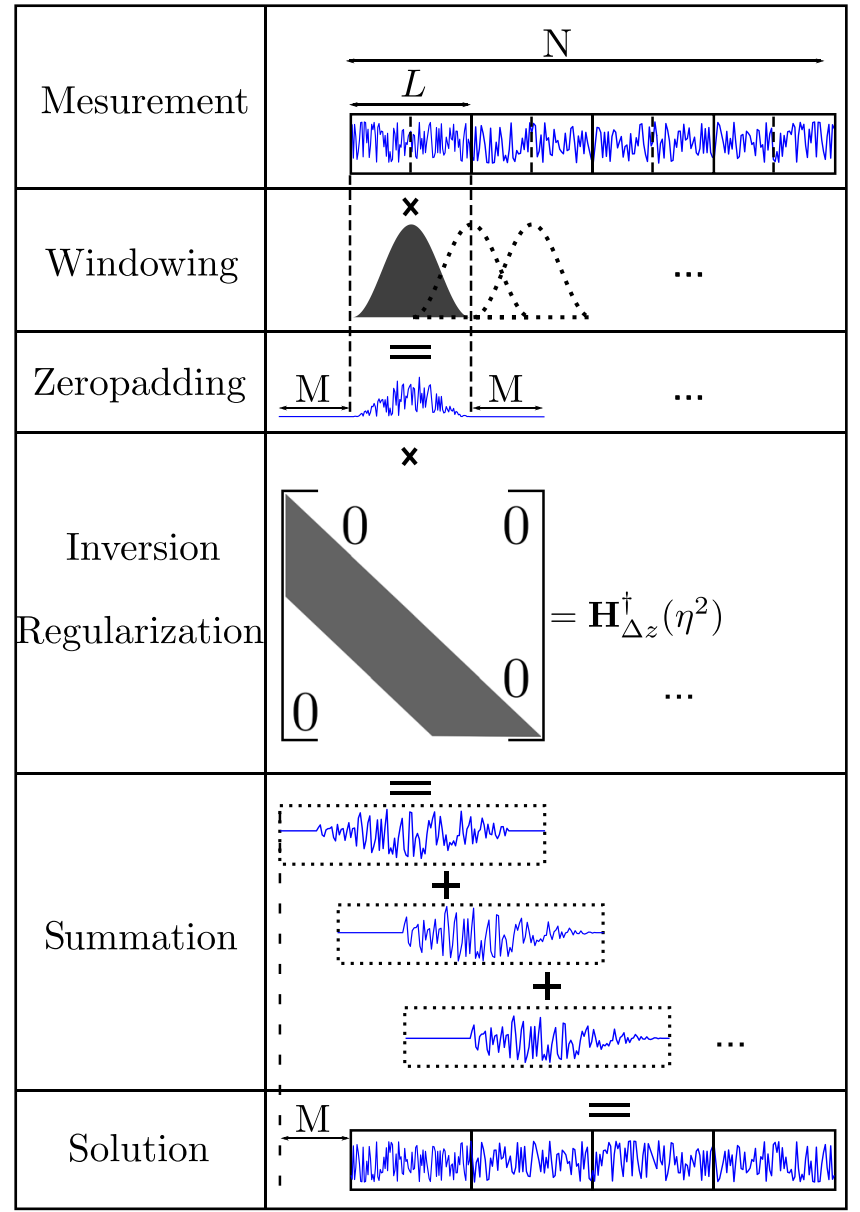

FIG. 2. (Color online) Batch deconvolution process applied to reconstruct long signals by RT-NAH method.

$$
\begin{aligned}
T_{1}^{i j} & =\frac{\left\langle p\left(x_{i}, y_{j}, z_{c}, t\right) \tilde{p}\left(x_{i}, y_{j}, z_{c}, t\right)\right\rangle}{\sqrt{\left\langle p^{2}\left(x_{i}, y_{j}, z_{c}, t\right)\right\rangle\left\langle\tilde{p}^{2}\left(x_{i}, y_{j}, z_{c}, t\right)\right\rangle}}, \\
T_{2}^{i j} & =\sqrt{\frac{\left\langle\tilde{p}^{2}\left(x_{i}, y_{j}, z_{c}, t\right)\right\rangle}{\left\langle p^{2}\left(x_{i}, y_{j}, z_{c}, t\right)\right\rangle}}
\end{aligned}
$$

where $\tilde{\mathbf{p}}^{2}\left(x_{i}, y_{j}, z_{c}, t\right)$ denotes the reference sound field in the time-space domain. The so-defined indicators are equal to 1 if the reconstruction is exact.

\section{B. Comparison with other regularization methods}

The performance of each method is analyzed on a large domain of observations. The position of the array varies from $5 \mathrm{~cm}$ to $50 \mathrm{~cm}$ and the signal to noise ratio (SNR) from $5 \mathrm{~dB}$ to $60 \mathrm{~dB}$. For each distance and SNR configuration, ten random samples have been recorded. Figures of merit $\left(1-T_{1}\right)$ and $\left(1-T_{2}\right)$ corresponding to the non-regularized (NR), LCV, GCV and BA solutions are plotted in Fig. 3. These indicators are averaged over the ten random samples and over the whole scan area. For readability reasons, indicators are set to an upper limit of 1 .

These results exhibit acceptable solutions when the array is close to the source, whatever the SNR. For distances above $0.1 \mathrm{~cm}$, the non-regularized solution becomes unstable and results given by GCV and LCV are also unsatisfactory: the value of indicator $T_{2}$ becomes very large, even if the phase reconstruction is satisfactory. To explain the reason why all methods are more sensitive at large backpropagation distances, the condition number is introduced as the ratio of the maximum to the minimum singular values, $K^{i j}=s_{\text {max }}^{i j} / s_{\text {min }}^{i j}$. Its value reflects the ill-posed severity of the problem. Its averaged value over the wavenumber is evaluated as a function of propagation distance in Fig. 4. For high wavenumber pairs, the condition number becomes high $[16.3 \mathrm{~dB}$ for a distance of $0.15 \mathrm{~m}$ for the pair $\left.\left(k_{x}, k_{y}\right)=(7,7)\right]$, which reflects the difficulty to reconstruct evanescent components at a distance larger than $0.1 \mathrm{~m}$ in our case. Based on non-regularized results, the presence of a tiny fraction of noise $(\mathrm{SNR}=60 \mathrm{~dB})$ is enough to destabilize the reconstructed sound field.

Finally, for the major part of the examined domain, the Bayesian regularization offers satisfactory results. The improvement provided by Bayesian regularization significantly increases with the severity of the ill-posed characteristic of the problem (large distance of back propagation, important SNR). Bayesian regularization thus offers the possibility to use RT-NAH for longer distances.

A reconstructed time-signal at position $(0.2,0.4) \mathrm{m}$ by the four methods using an array positioned at $10 \mathrm{~cm}$ from the source plane is shown in Fig. 5. This favourable case illustrates the quality of the reconstruction for all of the methods.

In contrast, Fig. 6 displays a severe case for the near-field holography as the distance of back-propagation is $40 \mathrm{~cm}$. One
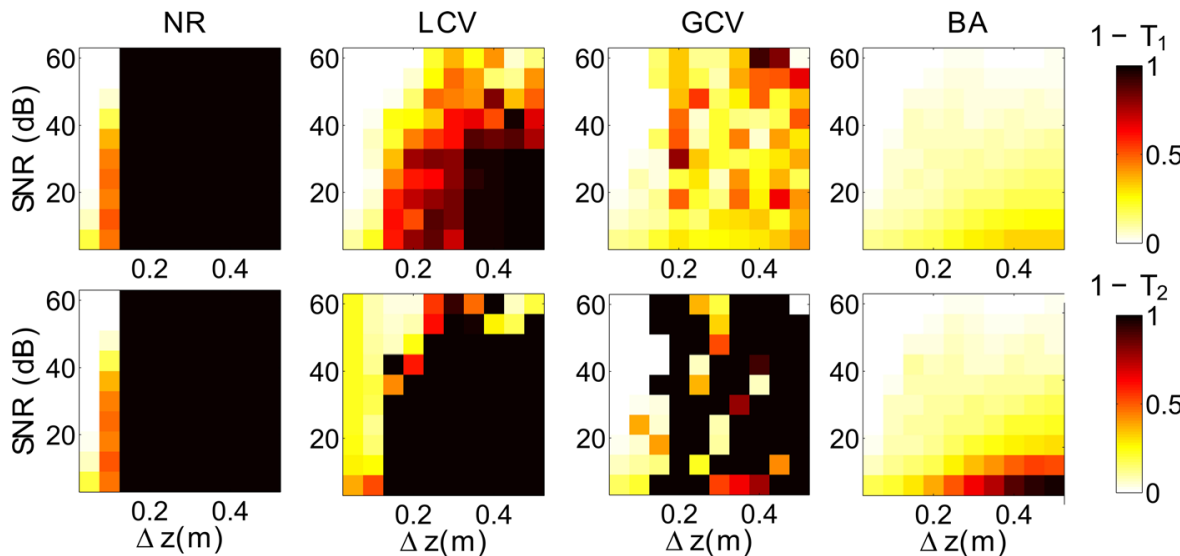

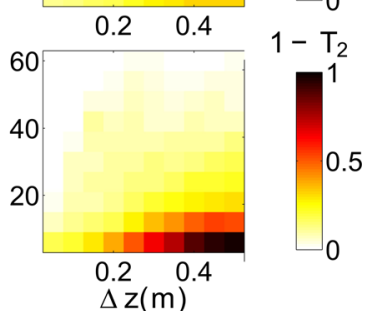

FIG. 3. (Color online) Indicators $1-T_{1}$ (above) and $1-T_{2}$ (below) averaged over ten random samples and over the space of calculation. From left to right: indicators for the nonregularized, $\mathrm{LCV}, \mathrm{GCV}$, and $\mathrm{BA}$ solutions. 


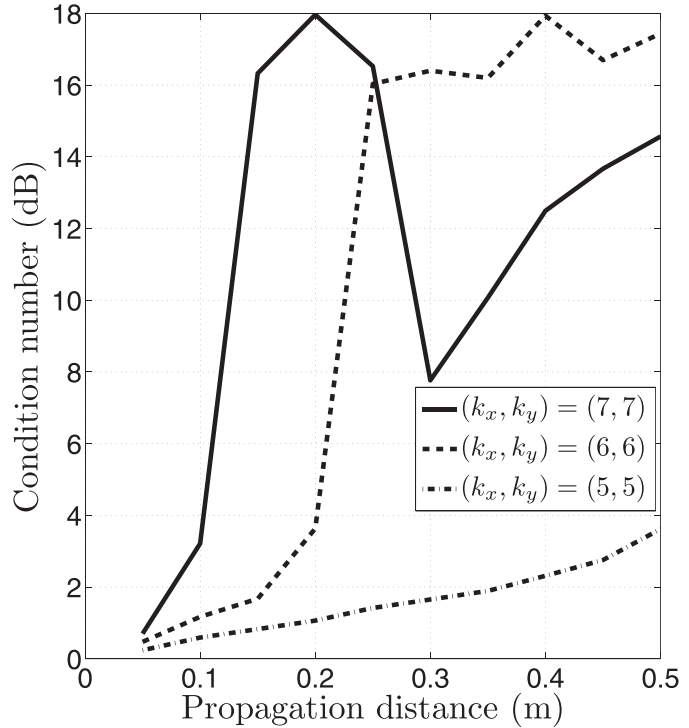

FIG. 4. Condition number as a function of distance for wavenumber pairs $(7,7),(6,6)$, and $(5,5)$.

can observe that the regularized solution diverges with the LCV and the GCV methods. This case illustrates the ill-posed behavior of the inverse problem: a little variation in the input causes a large variation at the output. Only the Bayesian regularization gives satisfactory results in this case.

The remarkably good results of the BA comes from the fact that the synthesized sound field from the source to the microphones fulfilled exactly the assumptions of the stochastic model on which the proposed Bayesian method is founded: the source and the noise have been randomly sampled from a complex Gaussian distribution. Conversely, the estimation of the regularization parameter by LCV or GCV does not come from an explicit stochastic model.

\section{Instantaneous regularization}

This subsection illustrates the batch deconvolution of a long non-stationary signal. The distance of retro-propagation is set to $\Delta_{z}=19 \mathrm{~cm}$ and a point source is positioned at $(0.2$, $0.4,0) \mathrm{m}$ generating a Morlet signal of central frequency $2000 \mathrm{~Hz}$. The SNR is set to $10 \mathrm{~dB}$ and 512 samples are used to describe the source signal. First, the signal has been reconstructed by the RT-NAH method using a matrix $\mathbf{H}_{\Delta z}$ of dimension $512 \times 512$. In this case, one single inversion is required and one single regularization parameter is evaluated for each of the $\left(k_{x}, k_{y}\right)$ pairs. For the same simulation configuration, batch deconvolution is applied as described in Sec. IIID. The measured signal is divided into 5 blocks of 128 samples and $50 \%$ overlap is considered. The results are shown in Fig. 7.

In batch deconvolution, the regularization parameter follows the evolution of the SNR: the amount of regularization is important when the acoustic signal is weak and low when the source conveys energy. Moreover, the proposed batch deconvolution method suppresses a limitation of RT-NAH concerning the length of the reconstructed time-signal. Figure 8 depicts the reconstruction of a non-stationary signal of $1 \mathrm{~s}$. The simulation parameters are the same as in the previous study case and 16000 samples have been reconstructed.

\section{EXPERIMENTAL RESULTS}

Experiments have been undertaken in order to confirm the simulation results. Reconstruction of parietal pressure
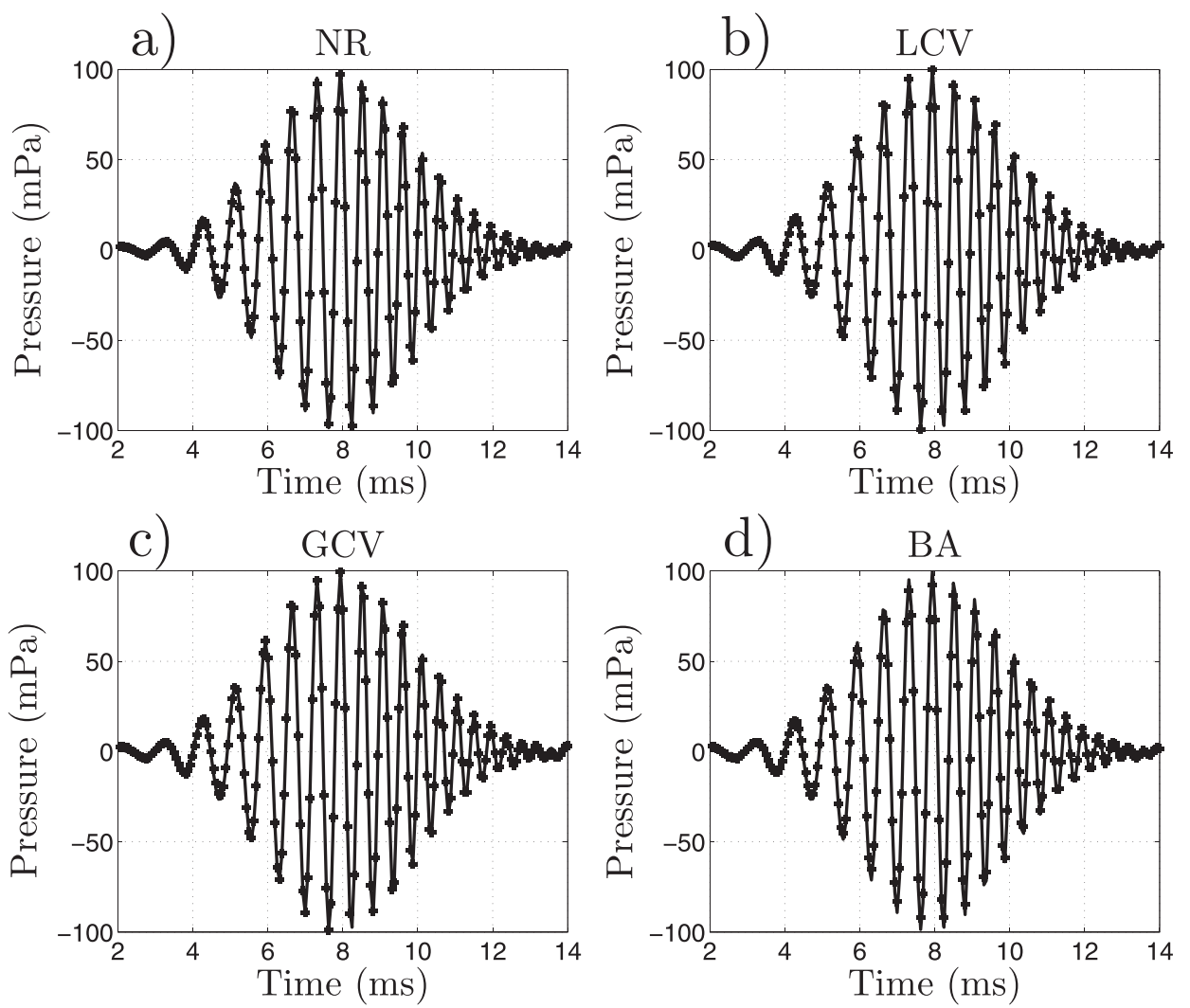

FIG. 5. Exact (solid line) and reconstructed (dotted line) signals without regularization (a), regularized by LCV (b), GCV (c), and BA (d) methods at $(0.2,0.4) \mathrm{m}$ for a SNR of $40 \mathrm{~dB}, z_{c}$ $=0.01 \mathrm{~m}$, and $\Delta z=0.1 \mathrm{~m}$. 

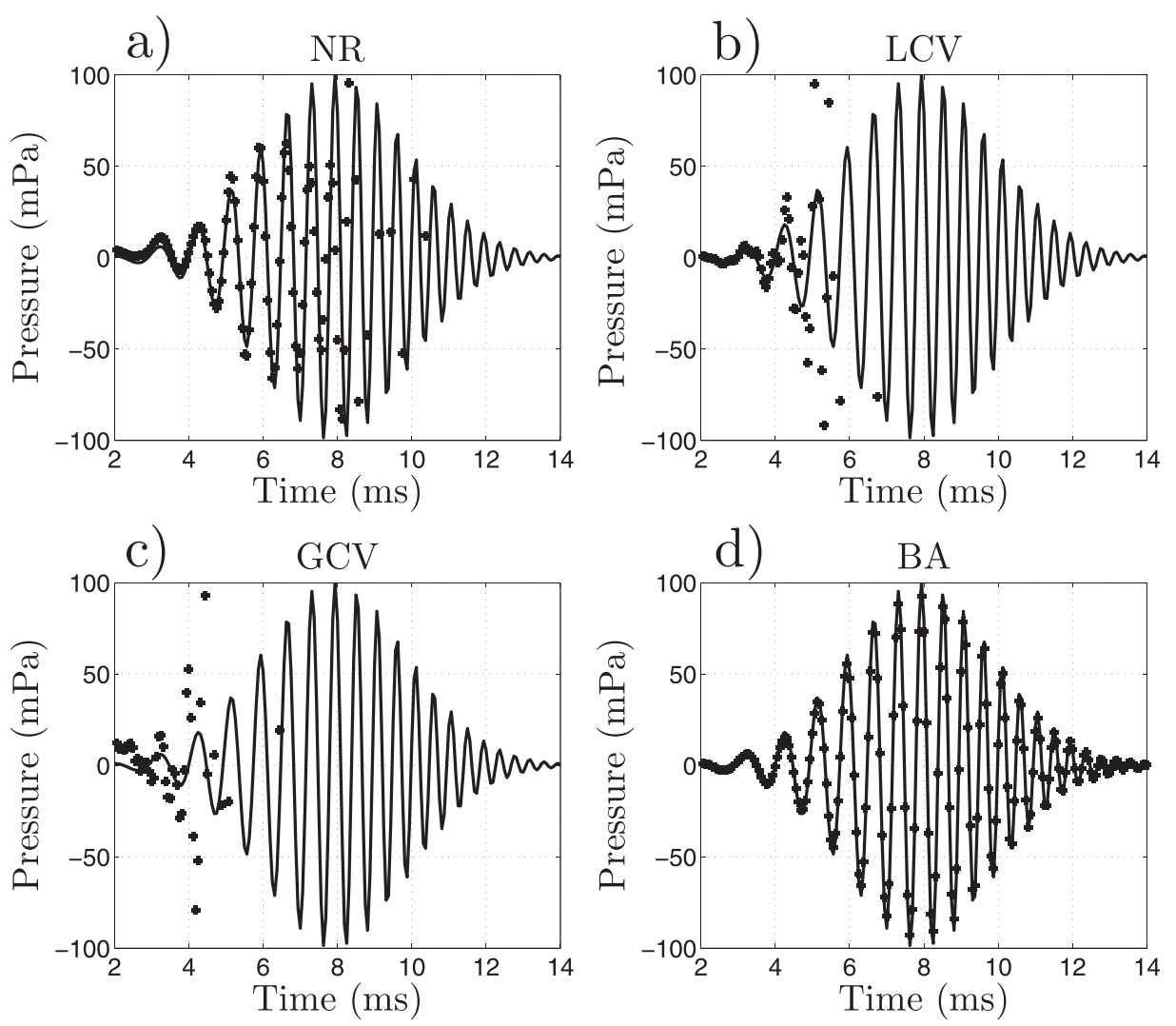

FIG. 6. Exact (solid line) and reconstructed (dotted line) signals without regularization (a), regularized by LCV (b), GCV (c), and BA (d) methods at $(0.2,0.4) \mathrm{m}$ for a SNR of $40 \mathrm{~dB}, z_{c}$ $=0.01 \mathrm{~m}$, and $\Delta z=0.4 \mathrm{~m}$. field on a steel plate is proposed to validate the instantaneous Bayesian regularization results. The steel plate has dimensions of $0.32 \mathrm{~m} \times 0.3 \mathrm{~m}$. It is mounted on a rigid structure and elastic straps are used to enable it to vibrate freely. The shaker excitation point is placed at $(0.41,0.18) \mathrm{m}$. Meanwhile a rigid rod maintains the contact between the plate and the shaker. The forced excitation signal is shown in Fig. 9.
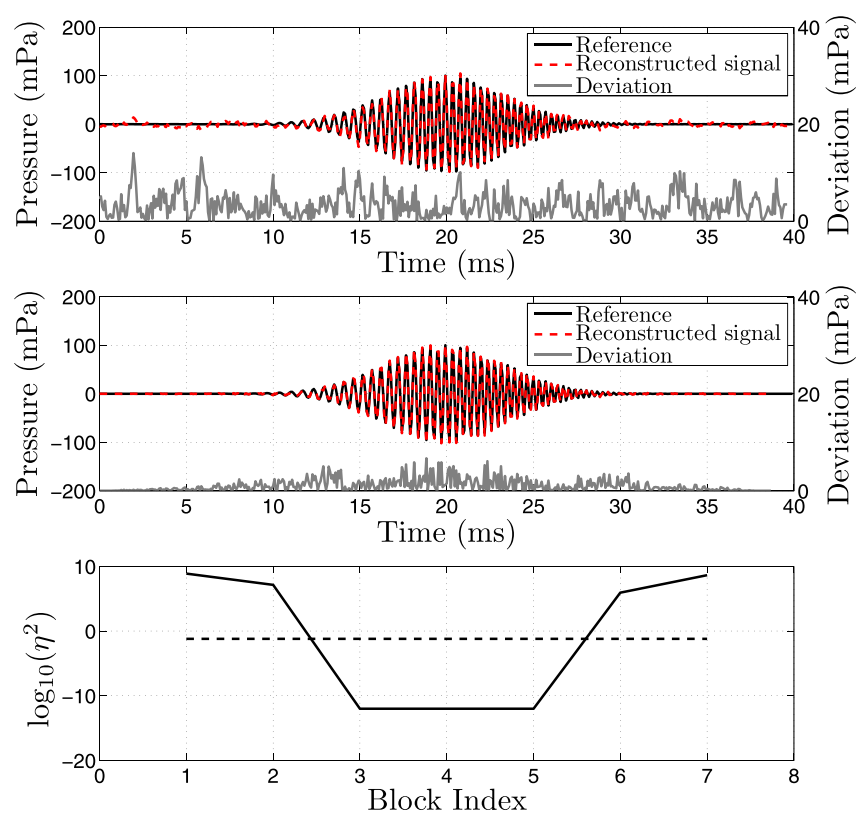

FIG. 7. (Color online) (a) Reconstructed signal at $(0.2,0.4,0.01) \mathrm{m}$ by global and (b) batch deconvolution. (c) Values of the regularization parameter in each block (averaged on wavenumbers) are shown in solid line and of the global regularization parameter in dotted line.
An impulse signal at $t=0 \mathrm{~ms}$ is set as the trigger to activate the acquisition. The signals are recorded at a sampling frequency of $25800 \mathrm{~Hz}$ providing 512 samples. The antenna, located $0.11 \mathrm{~m}$ from the plate, is composed of 49 microphones regularly-spaced by $0.1 \mathrm{~m}$, covering a total scan area of 0.7 $\times 0.7 \mathrm{~m} .^{2}$ The array's frequency bandpass is about $[490 \mathrm{~Hz}$,
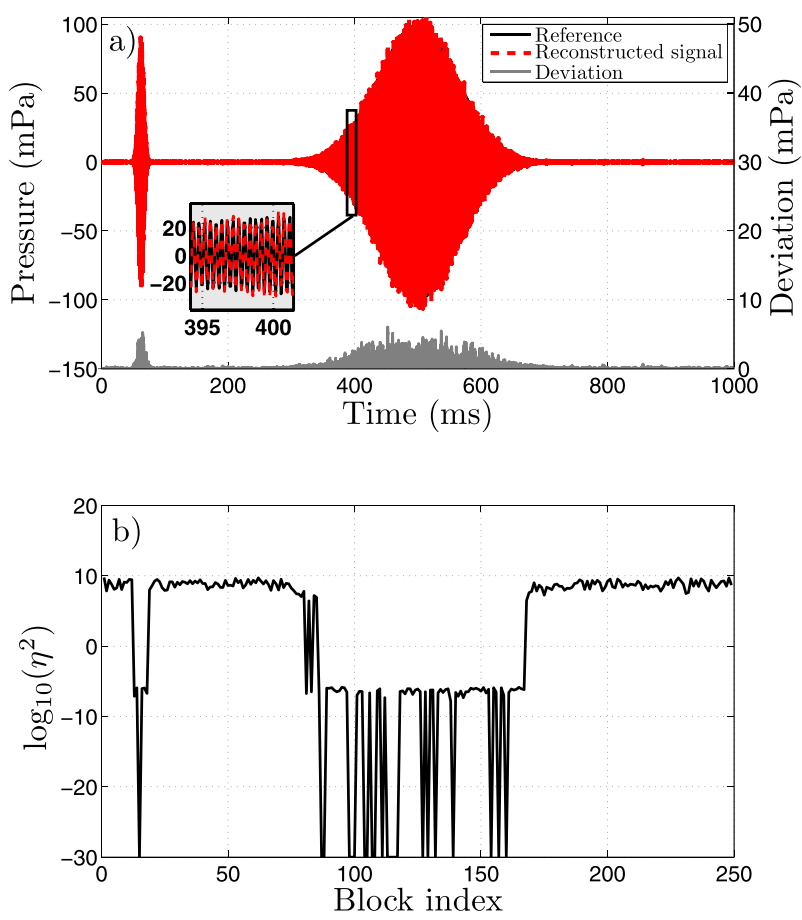

FIG. 8. (Color online) (a) Reconstructed signal at $(0.2,0.4,0.01) \mathrm{m}$ computed by batch deconvolution and (b) the corresponding instantaneous value of the regularization parameter. 


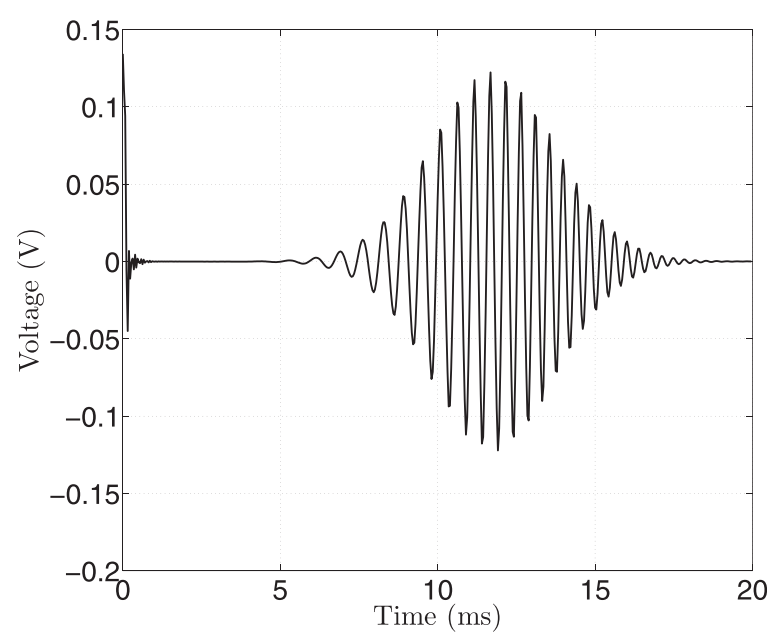

FIG. 9. Signal injected to the shaker.

$1770 \mathrm{~Hz}$. Since the array spans well the plate, no zero padding is operated on the space data. The entire set-up is shown in Fig. 10.

The batch deconvolution is applied on a block of 64 samples with $50 \%$ overlap, providing 15 blocks. The reconstruction results given by the four methods (NR, LCV, GCV, BA) are compared with the measured sound field at $z_{c}=0.01 \mathrm{~m}$ in Fig. 11, for a point close to the source excitation $(0.2 \mathrm{~m}, 0.2 \mathrm{~m})$. Even if the results provided by the Bayesian method under-estimate the maximum amplitude of the reference signal, it allows the recovery of the shape of the signal measured at $0.01 \mathrm{~m}$ whereas the other methods fail to do so.

Spatial reconstructions of the acoustic pressure fields provided by LCV, GCV, and BA regularizations are shown in Fig. 12 at time instants $t=7.6,10,12.1$, and $13.8 \mathrm{~ms}$.

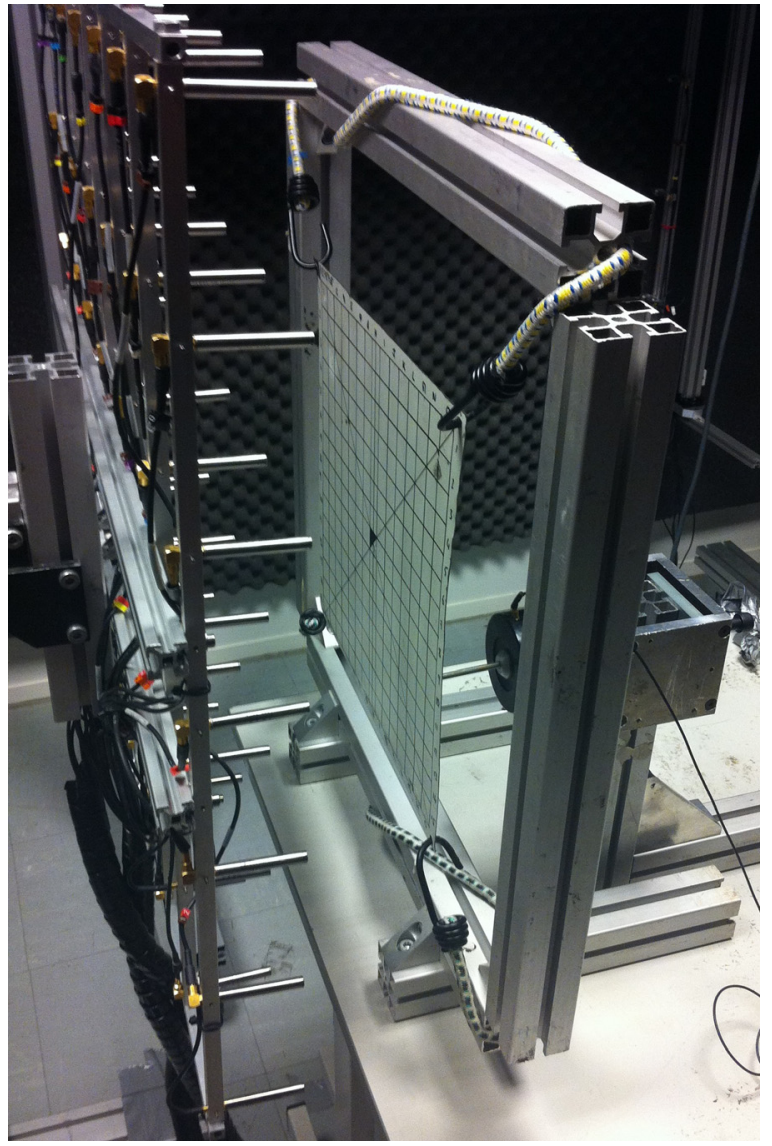

FIG. 10. (Color online) Photography of the experimental set-up.

Similar to the simulation results, Bayesian regularization provides more accurate results in terms of reconstructed levels and localization of sound sources. In fact, the side lobes present in the LCV and GCV results could lead the engineer
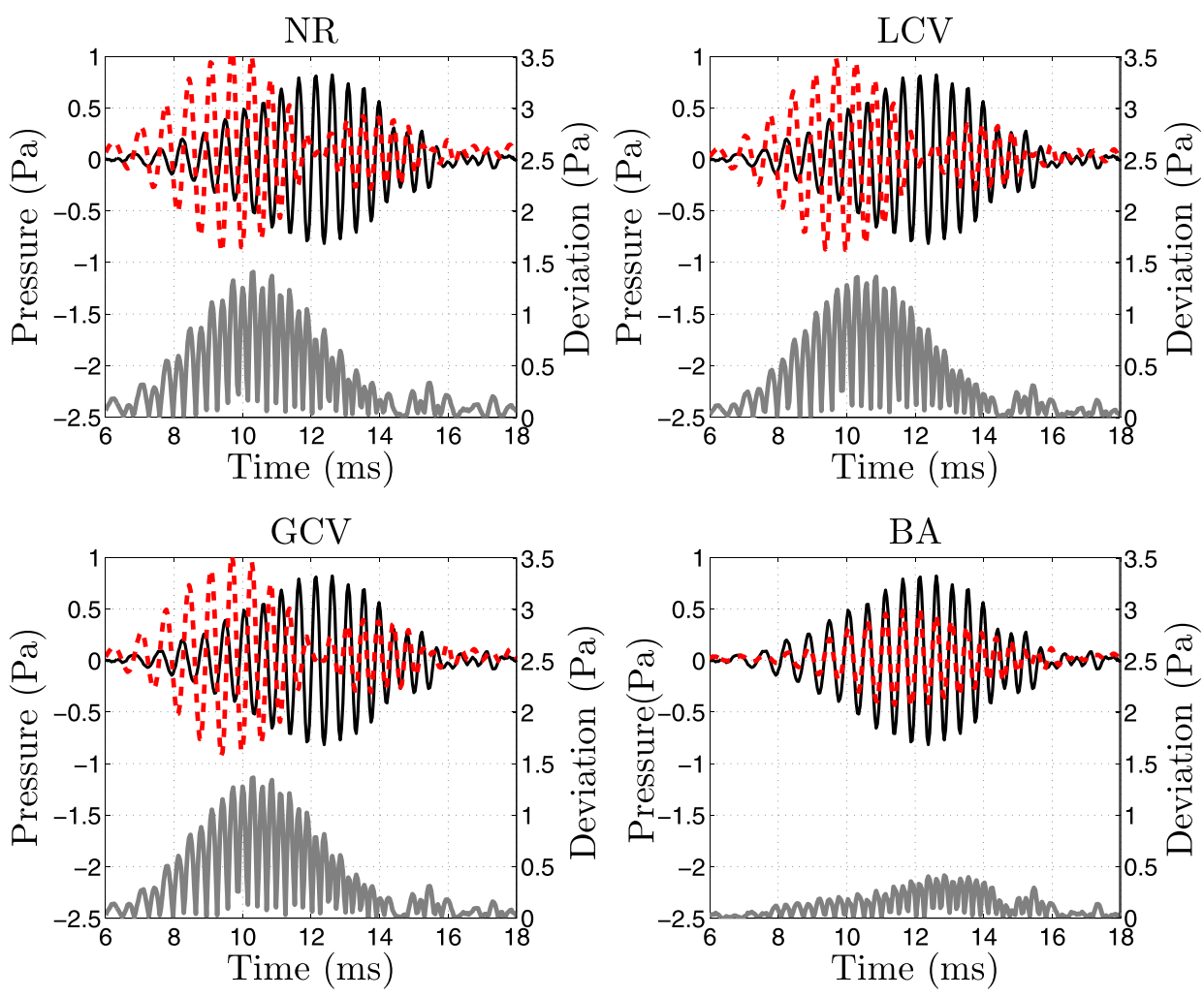

FIG. 11. (Color online) Pressure field measured at $(0.2 \mathrm{~m}, 0.2 \mathrm{~m}, 0.01 \mathrm{~m})$ (solid line) and reconstructed at $0.01 \mathrm{~m}$ from measurements at $0.11 \mathrm{~m}$ (a) without regularization (NR), by (b) LCV, (c) GCV, and (d) BA methods (red dotted line). The deviation between the reconstructed signal and the reference is shown in gray. 

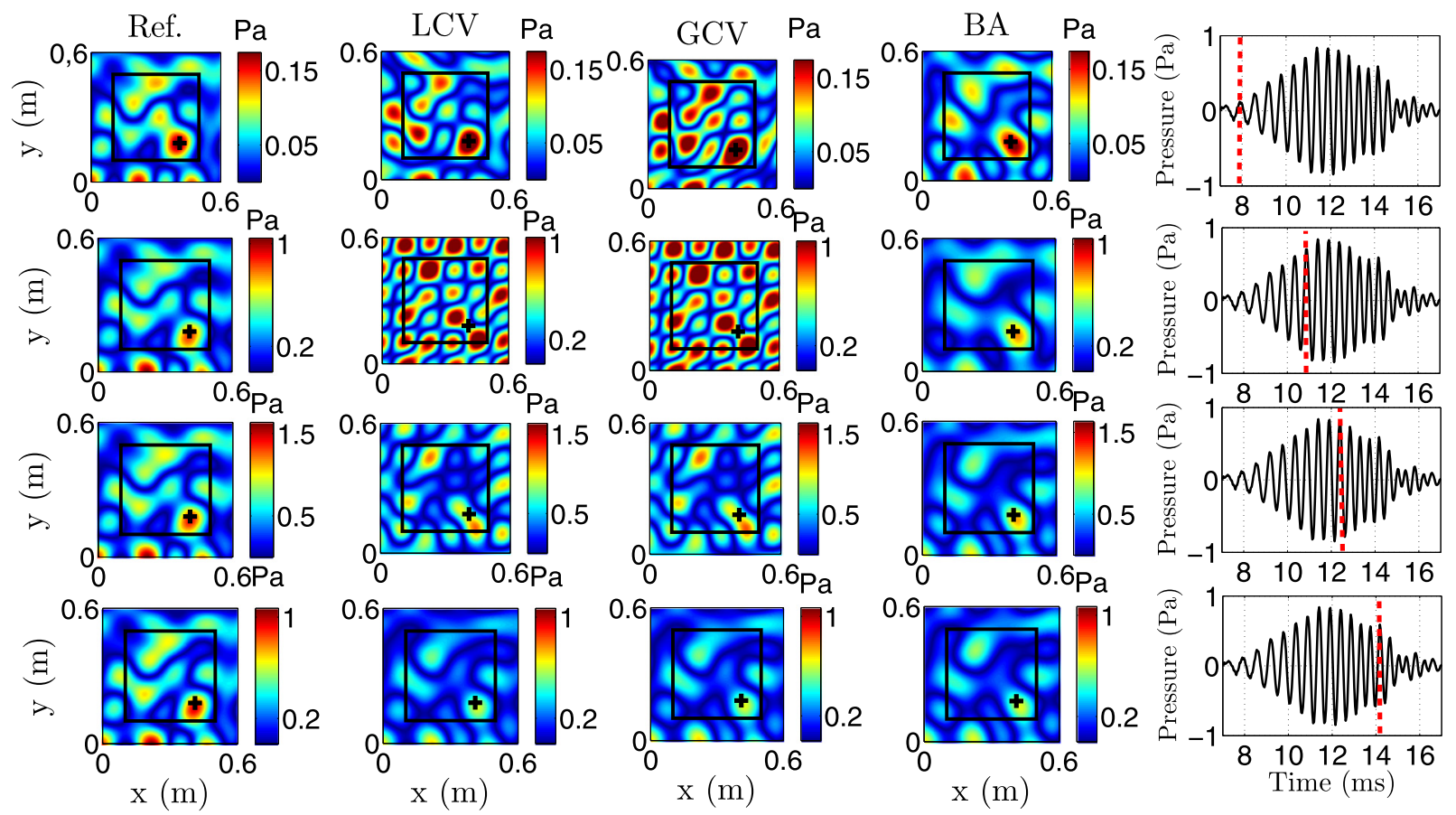

FIG. 12. (Color online) Spatial sound pressure field measured at $0.01 \mathrm{~m}$ (reference) and its reconstruction from measurements done at $0.11 \mathrm{~m}$ by the LCV, $\mathrm{GCV}$, and BA methods at time instants $t=7.6 \mathrm{~ms}, t=10 \mathrm{~ms}, t=12,1 \mathrm{~ms}$, and $t=13,8 \mathrm{~ms}$ (successively from top to bottom) identified by the dotted red line of the reference signal in the right column. The black rectangle represents the spatial limits of the steel-plate.

to erroneous acoustical diagnosis. The Bayesian reconstruction smooths the localization lobes but does not introduce spurious side lobes. Moreover, one can observe that the LCV and GCV methods tend to localize sources at the microphone positions (particularly at time instants $t=10$ and $12.1 \mathrm{~ms}$ ). This behavior typically reveals under-regularization.

Figure 13 shows the reconstructed signal at $(0.4,0.2) \mathrm{m}$ computed by one global block and computed by batch
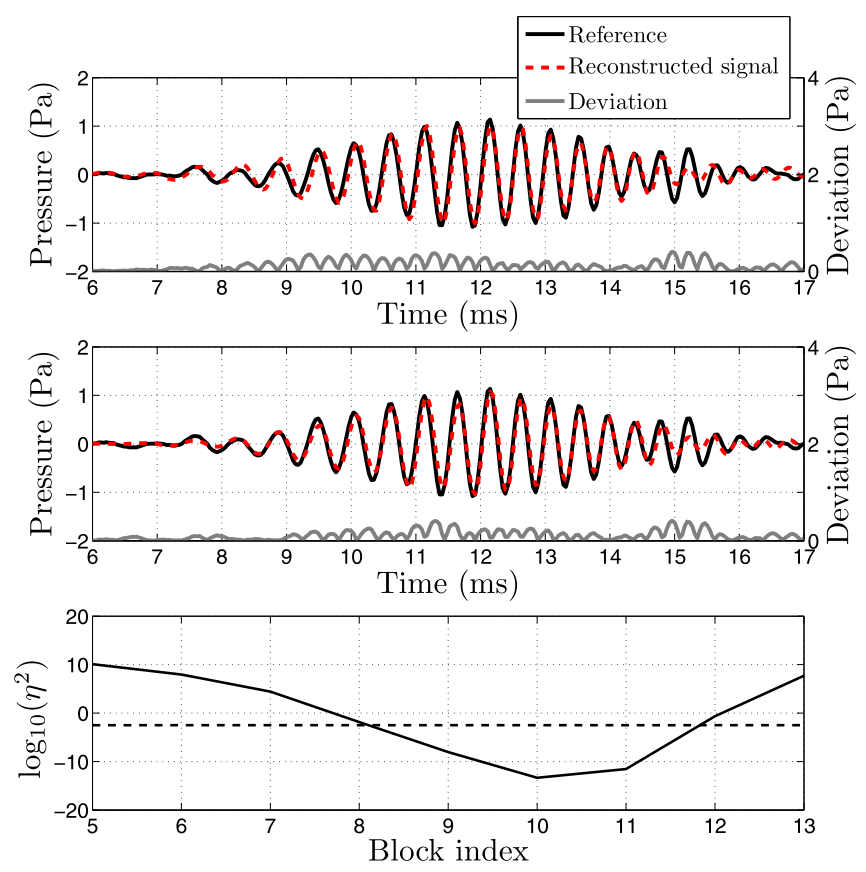

FIG. 13. (Color online) Exact (solid line) and reconstructed (red dotted line) signals at $(0.4,0.2) \mathrm{m}$ computed by (a) a global and (b) a batch deconvolution. (c) Regularization values averaged over the 49 pairs of wavenumbers for the global (dotted line) and the batch deconvolution (solid line). deconvolution. Similar to the numerical experiments, the instantaneous values of the regularization parameter follows the fluctuation of the energy of the source. However, here, the differences between the two processes are not as significant as in the numerical study. Only a better reconstruction of the first samples at time instants near $t=9 \mathrm{~ms}$ is noticed due to important gap between instantaneous regularization and the global one.

It has been noticed that experimental results underperform the numerical cases. This remark points out some limitations of RT-NAH. First, the test environment is not perfectly anechoic and reflections or modal behavior of the room may perturb the measurements. The direct model used in RT-NAH is built from a free-field assumption and the proposed approach of the regularization process is only efficient to tackle Gaussian background noise and not bias errors. Second, like NAH, RT-NAH decomposes the sound field into a plane wave basis. The proposed experimental case produces both plane waves and spherical waves to the array due to the relatively small size of the plate compared to the array. The decreasing amplitude of the spherical waves cannot be described by the plane wave basis. This observation could explain why the phase of the reconstructed sound field seems relatively well-resolved while the reconstructed amplitude under-estimates the reference.

\section{CONCLUSION}

Bayesian regularization has been introduced in the case of non-stationary source reconstruction using real-time nearfield acoustic holography. The working assumptions on the noise and source probability density functions (complex Gaussians) naturally lead to optimal solutions in the form of a Tikhonov regularized solution, together with a criterion to 
identify the regularization parameter. The performance of the latter has been compared to the LCV and the GCV methods. For a large domain of observations and according to a numerical study, Bayesian regularization is found to give more accurate results particularly when the problem becomes illposed. Moreover, it has been noted that updating the regularization parameter as a function of time is advantageous for highly non-stationary signals; the amount of regularization then directly follows the fluctuation of the SNR. Finally, in complement to numerical results, an experimental validation of the Bayesian regularization has been proposed. It reveals that Bayesian regularization allows the best reconstruction of the acoustic field generated by a steel plate in free field. From an engineering perspective, both its ease of implementation and its robustness with regard to noise disturbance in stationary and non-stationary conditions represent two strong advantages to use the Bayesian regularisation.

\section{ACKNOWLEDGMENTS}

This work was conducted in the framework of a CIFRE thesis between MicrodB, the LabEx CeLyA (Centre Lyonnais d'Acoustique), (ANR-10-LABX-60/ANR-11-IDEX-0007), and the Laboratoire d'Acoustique de l'Université du Maine (LAUM) (UMR CNRS-6613). We also would like to thank Florian Hughes for his help during his six-month placement in the MicrodB team.

${ }^{1}$ E. G. Williams, Fourier Acoustics: Sound Radiation and Nearfield Acoustical Holography (Academic, San Diego, 1999), pp. 89-114.

${ }^{2}$ J. Hald, "Time domain acoustical holography and its applications," Sound Vib. 35(2), 16-25 (2001).

${ }^{3}$ F. Deblauwe, J. Leuridan, J. L. Chauray, and B. Béguet, "Acoustic holography in transient conditions," in Proceedings of the Sixth International Congress on Sound and Vibration, Copenhagen, Denmark (July 1999), pp. 899-906.

${ }^{4}$ J. F. Blais and A. Ross, "Forward projection of transient sound pressure fields radiated by impacted plates using numerical Laplace transform," J. Acoust. Soc. Am. 125(5), 3120-3128 (2009).

${ }^{5}$ J. M. Attendu, J. F. Blais, and A. Ross, "Investigation of transient nearfield acoustical holography using temporal and spatial Laplace transforms," in Proceedings of the ICSV19 International Congress on Sound and Vibration, Vilnius, Lithuania (July, 2012), pp. 899-906.

${ }^{6} \mathrm{~S}$. F. Wu, "Reconstruction of transient acoustic radiation from a sphere," J. Acoust. Soc. Am. 117(4), 2065-2077 (2005)

${ }^{7} \mathrm{~S}$. F. Wu, "Reconstructing transient acoustic radiation from an arbitrary object with a uniform surface velocity distribution," J. Acoust. Soc. Am. 136(2), 514-524 (2014).

${ }^{8}$ M. R. Bai and J. H. Lin, "Source identification system based on the timedomain nearfield equivalence source imaging: Fundamental theory and implementation," J. Sound Vib. 307(1), 202-225 (2007).

${ }^{9}$ E. G. Williams, "Regularization methods for near-field acoustical holography," J. Acoust. Soc. Am. 110(4), 1976-1988 (2001).
${ }^{10}$ S. Paillasseur, J. H. Thomas, and J. C. Pascal, "Regularization for improving the deconvolution in real-time near-field acoustic holography," J. Acoust. Soc. Am. 129(6), 3777-3787 (2011).

${ }^{11}$ S. Paillasseur, "Développement des techniques d'holographie acoustique de champ proche temps réel pour l'analyse de sources de bruit fluctuantes" ("Development of real time nearfield acoustical holography techniques for analyzing fluctuating noise sources"), Ph.D. thesis, Université du Maine, Le Mans, France (2009), pp. 1-130.

${ }^{12}$ X. Z. Zhang, C. X. Bi, Y. B. Zhang, and L. Xu, "Transient nearfield acoustic holography based on an interpolated time-domain equivalent source method," J. Acoust. Soc. Am. 130(3), 1430-1440 (2011).

${ }^{13}$ X. Z. Zhang, C. X. Bi, Y. B. Zhang, and L. Xu, "Cubic spline interpolation-based time-domain equivalent source method for modeling transient acoustic radiation," J. Sound Vib. 332(22), 5939-5952 (2013).

${ }^{14}$ S. Pan and W. Jiang, "A hybrid approach to reconstruct transient sound field based on the free-field time reversal method and interpolated timedomain equivalent source method," J. Sound Vib. 333, 3625-3638 (2014).

${ }^{15}$ X. Z. Zhang, J. H. Thomas, C. X. Bi, and J. C. Pascal, "Reconstruction of nonstationary sound fields based on the time domain plane wave superposition method," J. Acoust. Soc. Am. 132(4), 2427-2436 (2012).

${ }^{16} \mathrm{~L}$. Geng, X. Z. Zhang, and C. X. Bi, "Reconstruction of transient vibration and sound radiation of an impacted plate using time domain plane wave superposition method," J. Sound Vib. 344, 114-125 (2015).

${ }^{17}$ J. H. Thomas, V. Grulier, S. Paillasseur, J. C. Pascal, and J. C. Le Roux, "Real-time near-field acoustic holography for continuously visualizing nonstationary acoustic fields," J. Acoust. Soc. Am. 128(6), 3554-3567 (2010).

${ }^{18}$ M. R. Bai, C. C. Chen, and J. H. Lin, "On optimal retreat distance for the equivalent source method-based nearfield acoustical holography," J. Acoust. Soc. Am. 129(3), 1407-1416 (2011).

${ }^{19}$ J. Antoni, "A Bayesian approach to sound source reconstruction: Optimal basis, regularization, and focusing," J. Acoust. Soc. Am. 131(4), 2873-2890 (2012).

${ }^{20}$ J. Idier, Bayesian Approach to Inverse Problems, 1st ed. (Wiley, New York, 2008), pp. 1-392.

${ }^{21}$ P. C. Hansen, Rank-Deficient and Discrete Ill-Posed Problems: Numerical Aspects of Linear Inversion (Society for Industrial and Applied Mathematics, Philadelphia, 1998), pp. 1-175.

${ }^{22}$ A. Pereira, J. Antoni, and Q. Leclère, "Empirical Bayesian regularization of the inverse acoustic problem," Appl. Acoust. 97, 11-29 (2015).

${ }^{23}$ M. Forbes, S. Letcher, and P. Stepanishen, "A wave vector, time-domain method of forward projecting time-dependent pressure fields," J. Acoust. Soc. Am. 90(5), 2782-2793 (1991).

${ }^{24}$ V. Grulier, S. Paillasseur, J. H. Thomas, J. C. Pascal, and J. C. Le Roux, "Forward propagation of time evolving acoustic pressure: Formulation and investigation of the impulse response in time-wavenumber domain," J. Acoust. Soc. Am. 126(5), 2367-2378 (2009).

${ }^{25} \mathrm{H}$. Cramér, Mathematical Methods of Statistics (PMS-9) (Princeton University Press, Princeton, 1999), pp. 1-575.

${ }^{26}$ P. M. Lee, Bayesian Statistics: An Introduction, 4th ed. (Wiley, Chichester, 2012), pp. 1-486.

${ }^{27}$ A. Pereira, "Imagerie acoustique en espace clos" ("Acoustic imaging in enclosed spaces"), Ph.D. thesis, INSA, Lyon, France (2013), pp. 1-173.

${ }^{28}$ Y. Kim and P. A. Nelson, "Optimal regularisation for acoustic source reconstruction by inverse methods," J. Sound Vib. 275(3), 463-487 (2004).

${ }^{29} \mathrm{Q}$. Leclère, “Acoustic imaging using under-determined inverse approaches: Frequency limitations and optimal regularization," J. Sound Vib. 321(3), 605-619 (2009). 